\title{
s \\ Detection of Brain Tumor Using Self Organizing Map With K-mean Algorithm
}

\author{
${ }^{1}$ Pradeep Singh Yadav $\&{ }^{2}$ Chandrahas Sahu \\ ${ }^{1}$ SSITM (Bhilai) , ${ }^{2}$ SSCET(Bhilai)
}

\begin{abstract}
The part of the image that has the tumor has more intensity in that portion and we can make our assumptions about the radius of the tumor in the image, these are the basic things considered in the algorithm. First of all some image enhancement and noise reduction techniques are used to enhance the image quality, after that some morphological operations are applied to detect the tumor in the image. The morphological operations are basically applied on some assumptions about the size and shape of the tumor and in the end the tumor is mapped onto the original gray scale image with 255 intensity to make visible the tumor in the image. The algorithm has been tried on a number of different images from different angles and has always given the correct desired result.
\end{abstract}

Index Terms- object recognition, characteristic vector, edge tracing, feature detection

\section{INTRODUCTION}

The purpose of our project is to identify a tumor from a given MRI scan of a brain using digital image processing techniques. In this self organizing map is used for dividing the whole image into number of cluster, than after K-mean algorithm is used for clustering of cluster.

\section{TUMOR}

A tumor or tumor is the name for a neoplasm or a solid lesion formed by an abnormal growth of cells (termed neoplastic) which looks like a swelling .Tumor is not synonymous with cancer. A tumor can be benign pre-malignant or malignant, whereas cancer is by definition malignant.

\section{TYPES OF TUMOR:}

BENIGN TUMOR: A benign tumor is a tumor that lacks all three of the malignant properties of a cancer. Thus, by definition, a benign tumor does not grow in an unlimited, aggressive manner, does not invade surrounding tissues, and does not spread to non-adjacent tissues (metastasize). Common examples of benign tumors include moles and uterine fibroids.
MALIGNAN : Malignancy (from the Latin roots mal- = "bad" and -ignis = "fire") is the tendency of a medical condition, especially tumors, to become progressively worse and to potentially result in death. It is characterized by the properties of anaplasia, invasiveness, and metastasis. Malignant is a corresponding adjectival medical term used to describe a severe and progressively worsening disease. The term is most familiar as a description of cancer.

PREMALIGNANT:A precancerous condition (or premalignant condition) is a disease, syndrome, or finding that, if left untreated, may lead to cancer. It is a generalized state associated with a significantly increased risk of cancer.

\section{MRI}

Magnetic resonance imaging (MRI), or nuclear magnetic resonance imaging (NMRI), is primarily a medical imaging technique used in radiology to visualize detailed internal structure and limited function of the body. MRI provides much greater contrast between the different soft tissues of the body than computed tomography (CT) does, making it especially useful in neurological (brain), musculoskeletal, cardiovascular, and oncological (cancer) imaging. Unlike CT, MRI uses no ionizing radiation. Rather, it uses a powerful magnetic field to align the nuclear magnetization of (usually) hydrogen atoms in water in the body. Radio frequency (RF) fields are used to systematically alter the alignment of this magnetization. This causes the hydrogen nuclei to produce a rotating magnetic field detectable by the scanner. This signal can be manipulated by additional magnetic fields to build up enough information to construct an image of the body.

\section{METHODOLOGY}

The part of the image containing the tumor normally has more intensity then the other portion and we can assume the area, shape and radius of the tumor in the image. We have used these basic conditions to detect tumor in our code and the code goes through th following steps: we present a new efficient edge tracing method, which was developed to find characteristic vector for the black-and-white images. The previously 
proposed edge tracing methods have two main disadvantages. These are: The edge detection of only one object in case of more than one object in a numerical image and investigating only outer contours not inner contours.

\section{FIGURES}

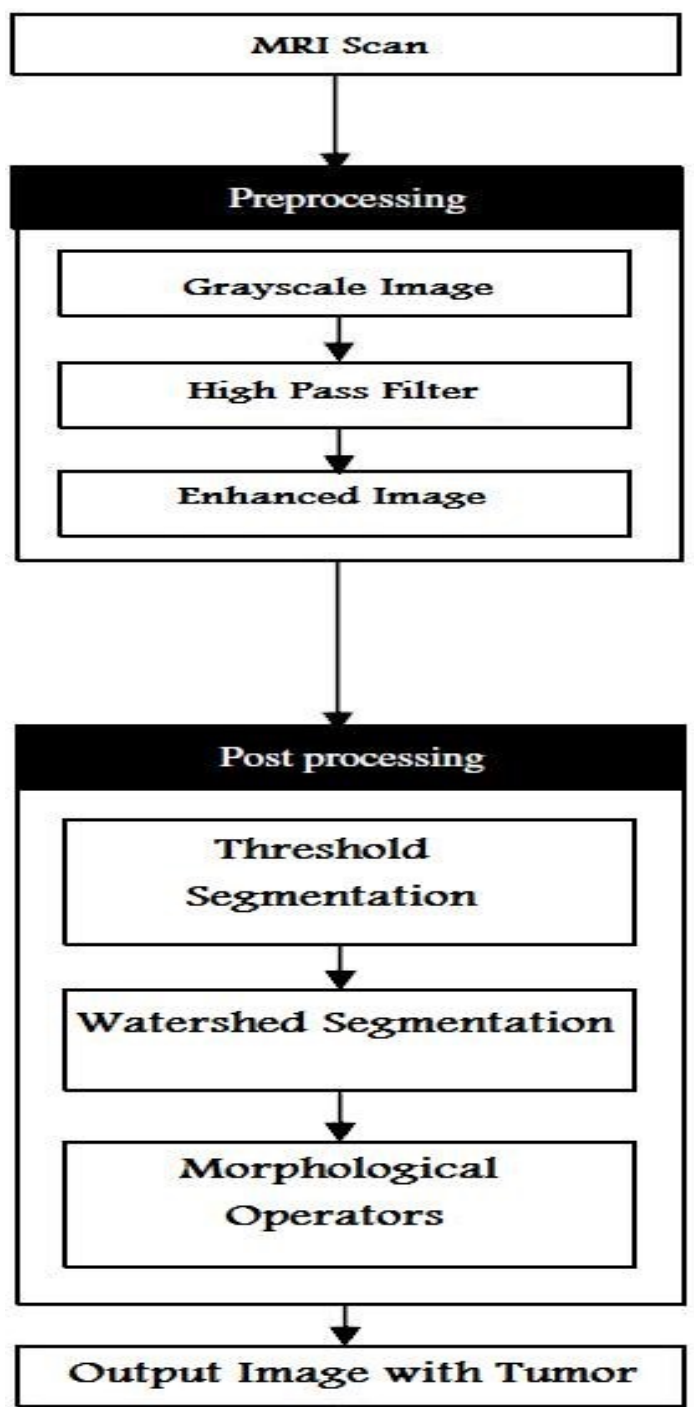

\section{PREPROCESSING}

In processing the following different steps are followed:-

THRESHOLD SEGMENTATION: Segmentation is done on basis of a threshold, due to which whole image is converted into binary image. Basic matlab commands for thresholding are used for this segmentation.

WATERSHED SEGMENTATION: It is the best method to segment an image to separate a tumor but it suffers from over and under segmentation, due to which we have used it as a check to our output. We have not used watershed segmentation on our input, rather it is only used on our output to check of the result is correct or not and it give the correct answer every time as is shown below.

\section{Morphological Operators:}

After that some morphological operations are applied on the image after converting it into binary form. The basic purpose of the operations is to show only that part of the image which has the tumor that is the part of the image having more intensity and more area then that specified in the strel command.

The basic commands used in this step are strel, imerode and imdilate.

\section{Imerode():}

It is used to erode an image.

Imdilate():

It is used to dilate an image

\section{OUTPUTS/RESULTS}

We have mapped the resultant tumor image onto the original grayscale image for presentation purposes
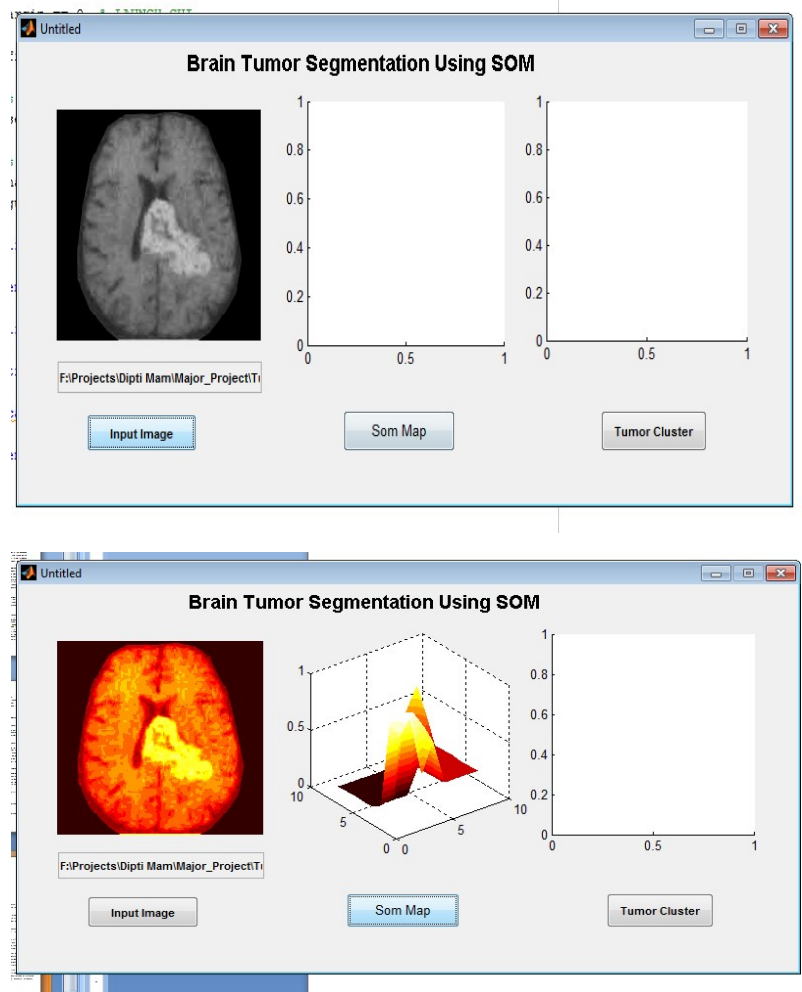


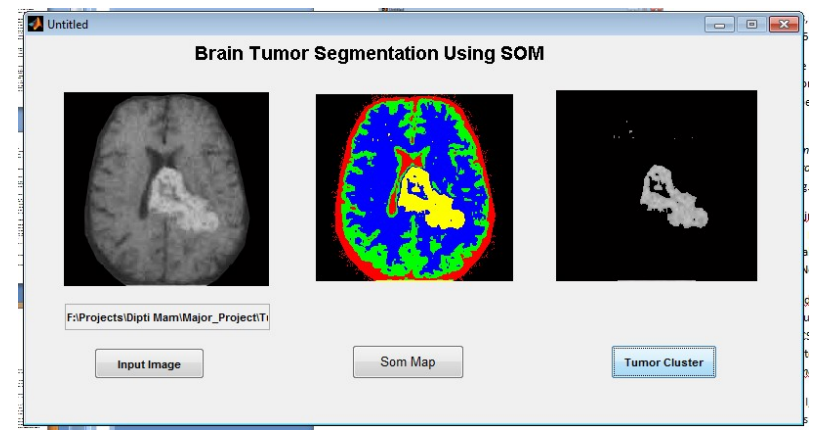

\section{REFERENCES}

[1] Bonavita S, Di Salle F, Tedeschi D., Proton MRS in neurological disorders, European Journal of Radiology, No.31, 1999, pp.30-125.

[2] Baik H, Choe B, Lee H, Suh T, Son B, Lee J., Metabolic Alterations in Parkinson's Disease after halamotomy, as Revealed by $1 \mathrm{H}$ MR Spectroscopy, Korean J Radiol, Vol.3, No.3, 2002, pp.180-9.
[3] Metin Akay, Time Frequency and Wavelets in Biomedical Signal Processing, IEEE Press Series on Biomedical Engineering.

[4] Starčuk Z, Starčuk jr, Horký J., 'Baseline' problems in very short echo-time proton MR spectroscopy of low molecular weight metabolites in the brain, Measurment Science Review, Vol.1, No.1, 2001, pp.17-20.

[5] Weber-Fahr W, Ende G, Braus D, Bachert P, Soher J, Henn F, Buchel C., A Fully Automated Method for Tissue Segmentation and CSFCorrection of Proton MRSI Metabolites Corroborates Abnormal Hippocampal NAA in Schizophrenia, NeuroImage, Vol.16, 2002, pp.49-60.

[6] Axelson D, Bakken I, Gribbestad I, Ehrnholm B, Nilsen G, Aasly J., Applications of Neural Network Analyses to In Vivo 1H Magnetic Resonance Spectroscopy of Parkinson Disease Patients, Journal of Magnetic Resonance Imaging, Vol.16, 2002, pp.13-20. 